\title{
Not the same old hindsight bias: Outcome information distorts a broad range of retrospective judgments
}

\author{
AMY BRADFIELD \\ Bates College, Lewiston, Maine \\ and \\ GARY L. WELLS \\ Iowa State University, Ames, Iowa
}

\begin{abstract}
The hindsight bias (e.g., Fischhoff, 1975) illustrates that outcome information can make people believe that they would have (or did) predict an outcome that they would not (or did not) actually predict. In two experiments, participants $(N=226)$ made a prediction immediately before receiving outcome information. Therefore, participants could not distort or misremember their predictions to make them align with the outcome information. In both experiments, participants distorted their reports of how certain they recalled having been in their prediction, how good of a basis they had for making the prediction, how long they took to make the prediction, and so forth. Experiment 2 showed that these effects were diminished when participants engaged in private thought about the upcoming questions prior to receiving outcome information, suggesting that the effect is not due to impression management concerns.
\end{abstract}

A robust psychological phenomenon known as the hindsight bias or "knew it all along" effect provides a framework for determining how people respond to outcome information (Fischhoff, 1975; Fischhoff \& Beyth, 1975; Wood, 1978). ${ }^{1}$ In typical demonstrations of the hindsight bias, people learn outcome information and then indicate what they would have predicted had they not been given the outcome information (e.g., Fischhoff's [1977] hypothetical paradigm). The hindsight bias exists when people overestimate their ability to have predicted the outcome. The hindsight bias also exists when people misremember earlier predictions so that they conform with outcome information (e.g., Fischhoff's [1977] memory paradigm). The typical hindsight paradigms do not account for situations in which people make predictions, receive outcome information, and accurately recall their prior predictions. The present research is designed to address just such a situation. In doing so, the experiments presented below address the effect of outcome information on reports of the decision processes that accompany a prediction rather than on reports of the prediction itself.

We thank Gina Buse, Nick Carnagey, Josh Fuller, Lindsay Hickle, John Neal, and Amy Schwering for help in collecting data. We thank Vesna Hampel and Luke Douglass for help in creating the experimental materials. We thank Luke Douglass, Dawn McQuiston, Michael Sargent, and Rebecca White for comments on earlier drafts of this article, which is based on a dissertation by A.B., under the supervision of G.L.W. Correspondence concerning this article should be addressed to A. Bradfield, Department of Psychology, Bates College, 4 Andrews Road, Lewiston, ME 04240 (e-mail: abradfie@bates.edu).

\section{Research on the Hindsight Bias}

The hindsight bias appears in two related experimental paradigms. For example, Fischhoff (1977) had people answer general knowledge questions (e.g., "Absinthe is [a] a precious stone or [b] a liqueur") in one of three groups. In the memory group, people answered questions, received the correct answers, and later recalled their responses. In the reliability group, people answered questions and then later recalled their responses (without knowledge of the correct answers). In the hypothetical group, people received information about the correct answers and then indicated how they would have answered the questions, had they not known the correct answers. People in the hypothetical group overestimated how much they would have known, compared with the reliability group's original responses, a phenomenon Fischhoff termed the "I knew it all along" effect. Fischhoff (1977) also observed a bias in the memory group. People who misremembered their earlier judgments did so in such a way that their recalled judgments were closer to the correct answer than were their original judgments. Therefore, there are two situations in which people display the hindsight bias. In the first, outcome information distorts people's estimates of how they would have answered, had they not known the outcome information (i.e., Fischhoff's hypothetical group). In the second, outcome information distorts people's recall of prior judgments (i.e., Fischhoff's memory group). Early research demonstrated that the hindsight bias was not a function of misinterpreted instructions (i.e., as a display of current knowledge instead of retrospective judgments; Fischhoff \& Beyth, 1975) or demand characteristics (Wood, 1978). 
Since Fischhoff's initial experiments, the hindsight bias has been demonstrated many times in both memory and hypothetical experimental paradigms (see metaanalysis by Christensen-Szalanski \& Willham, 1991; for a review, see Hawkins \& Hastie, 1990). In many of these experiments, people make judgments about uncertain outcomes rather than answer questions about general knowledge. For example, the hindsight bias has been demonstrated in evaluations of sexual assault victims (Carli, 1999; Janoff-Bulman, Timko, \& Carli, 1985), football games (Roese \& Maniar, 1997), jury decision-making (e.g., Casper, Benedict, \& Kelly, 1988; Hastie, Schkade, \& Payne, 1999; Robbennolt \& Sobus, 1997; Stallard \& Worthington, 1998), "gustatory judgments" (Pohl, Schwarz, Sczesny, \& Stahlberg, 2003), gambling behavior (Baboushkin, Hardoon, Derevensky, \& Gupta, 2001), competitive team decision making (Louie, Curren, \& Harich, 2000), determinations of therapist negligence (LaBine \& LaBine, 1996), economic expectations (Hölzl, Kirchler, \& Rodler, 2002), predictions of the likelihood of violent behavior (Cannon \& Quinsey, 1995), and medical diagnoses (Arkes, Wortmann, Saville, \& Harkness, 1981).

\section{Explanations for the Hindsight Bias}

There are two categories of explanations for the hindsight bias: automatic and motivational. If the hindsight bias is an automatic phenomenon, biased judgments occur outside of awareness. In Fischhoff's (1975) explanation of the hindsight bias, it was an automatic phenomenon; outcome information automatically altered people's recollections of the past, quickly and permanently supplanting previous knowledge, a process Fischhoff termed creeping determinism. The conceptualization of the hindsight bias as an automatic phenomenon has been supported in subsequent research (see, e.g., Hawkins \& Hastie, 1990; Hell, Gigerenzer, Gauggel, Mall, \& Müller, 1988; Hoffrage, Hertwig, \& Gigerenzer, 2000; Pohl, 1998; Pohl \& Hell, 1996).

If the hindsight bias is a motivational phenomenon, biased judgments are the result of people's desire to present themselves favorably. For example, suggesting that you "knew it all along" is an excellent way to present an image of yourself as a knowledgeable person or a good decision-maker. Indeed, Campbell and Tesser (1983) demonstrated that the magnitude of the hindsight bias was positively correlated with scores on a measure of social desirability. In another demonstration, people playing a market simulation game displayed the hindsight bias only when the outcome for their team was favorable (i.e., the firm's performance improved), suggesting that people claim to have "known it all along" when doing so enhances their image as a successful decision maker (Louie et al., 2000, see also, e.g., Hölzl et al., 2002). In general, however, support for motivational explanations of the hindsight bias is weak (e.g., Christensen-Szalanski \& Willham, 1991; Pohl \& Hell, 1996). For example, Fischhoff (1977) encouraged participants to "work hard" to recall their earlier judgments, making it clear that hindsight-free judgments were desirable. Even then, he found strong evidence of the hindsight bias (see also Sharpe \& Adair, 1993). More recently, Pohl and Hell (1996) gave participants feedback about their responses and instructed them to recall their original answers. After answering the same questions three times, participants were still unable to produce responses that eliminated the hindsight bias.

\section{Overview of the Current Research}

As noted earlier, existing theories of the hindsight bias cannot answer questions about how outcome information might affect a broad range of retrospective judgments related to a decision or prediction that people accurately recall. Determining whether such distortions occur is both practically and theoretically important. It is practically important because observers often use retrospective reports to evaluate the accuracy of another person's decision. If outcome information distorts retrospective reports of confidence or other judgments, observers will base assessments of accuracy on faulty information. And it is theoretically important to determine how broadly outcome information affects retrospective reports when people cannot misremember a previous decision. Recent research suggests that the hindsight bias is the result of inferences made from feelings of confidence or perceptual fluency (Werth \& Strack, 2003). The present research adds to that analysis by asking whether outcome information can distort the judgments from which these inferences are made. This research will add to our understanding of the hindsight bias, not by replicating the classic paradigm, but by testing the boundaries of situations in which outcome information distorts retrospective judgments. In order to test those boundaries, the experimental paradigm presented here differs from what is typical in hindsight research.

In the usual hindsight paradigm, people are given outcome information and asked what they would have predicted (the hypothetical paradigm) or people make a prediction, are told the correct answer, and asked to recall their prediction (memory paradigm). In both cases, the dependent measure of interest is people's reports of what they would (or did) predict. The present paradigm asks people to make a prediction and then immediately tells them that their prediction was correct or incorrect. Hence, with the present paradigm, there is no opportunity for people to mistakenly believe that they would have predicted the outcome or to mistakenly recall that they correctly predicted the outcome, which is the usual way of assessing the hindsight bias. Instead, the research question concerns what happens to people's thoughts when the outcome information confirms or disconfirms their predictions by asking about the information and the processes used to make the prediction.

The two experiments presented here examine the effects of outcome information after people make decisions about an observed romantic relationship. In both experiments, participants watched a short video of a cou- 
ple interacting and made a decision about the couple's relationship. After making their decision, participants heard bogus confirming or disconfirming feedback (i.e., outcome information) about the accuracy of their decision. Participants then answered an extensive set of questions instructing them to recall aspects of their decision that occurred before feedback was given.

Experiment 1 was designed to investigate whether randomly determined feedback about the accuracy of a decision would affect participants' judgments about the processes involved in their decision. These judgments were made under conditions in which participants could not alter their beliefs about whether or not they had made the correct decision. It was predicted that participants who received confirming feedback would inflate their retrospective reports, compared with participants who received disconfirming feedback. For example, it was predicted that participants' judgments about how confident they were at the time of their decision would be higher among those who received confirming feedback than among those who received disconfirming feedback. Similarly, it was predicted that participants who received confirming feedback would report that the decision was easier, took less time, and that they had a better basis for their decision, compared with participants who received disconfirming feedback. This prediction was based on the assumption that participants do not think about the judgments before reading the questionnaire. Because the questionnaire is presented after feedback is given, participants can only answer the questions in light of the information they have received about their accuracy. Therefore, participants who hear that their decision was correct might assume that they were confident at the time of their decision, given that their decision was correct. Similarly, participants who receive confirming feedback might claim that their decision was easy and was made quickly, compared with participants who receive disconfirming feedback. Experiment 2 was designed to test whether the effects observed in Experiment 1 were indeed due to this failure to consider the judgments assessed in the dependent measures questionnaire before hearing feedback. Specific predictions for Experiment 2 are discussed later.

\section{EXPERIMENT 1}

The present work presents an interpersonal judgment paradigm with three phases. In the first phase, participants viewed an event (a videotape of a couple at a counseling session) and then made a decision about that event (i.e., which person committed suicide or had an affair). In the second phase, participants were randomly assigned to hear confirming or disconfirming feedback about the accuracy of their decision. In the third phase, participants answered questions about the event and the processes involved in their decision. There was enough ambiguity in the event to ensure that both confirming and disconfirming feedback were credible, regardless of which decision the participants made. As noted above, it was hypothesized that participants who received confirming feedback about their decision would inflate their retrospective judgments, compared with participants who received disconfirming feedback. There were no hypotheses concerning the scenario (affair vs. suicide) about which participants made a decision.

\section{Method}

Participants and Design. The participants $(N=98 ; 64$ female $)$ were undergraduate psychology students at Iowa State University. They were given extra credit in their psychology classes in exchange for their participation. The participants watched a video in which a couple was interacting. After watching the video, the participants were instructed to make one of two decisions. Through random assignment, half of the participants heard that one member of the couple committed suicide shortly after the tape was made; they were instructed to indicate which person they thought committed suicide. The other half of the participants heard that one member of the couple had an affair shortly after the tape was made. The participants indicated which person they thought had an affair. After making their decision, all participants were randomly assigned to hear confirming or disconfirming feedback about the accuracy of their decision. The resulting design was a 2 (suicide scenario vs. affair scenario) $\times$ 2 (confirming feedback vs. disconfirming feedback) fully randomized, between-participants factorial.

Materials and Procedure. The participants arrived at the experiment location in pairs. After completing a consent form, the participants were told that they would watch a short (5-min) video of a man and a woman interacting. In the consent form, the participants were warned that, “. . .some people may experience mild feelings of discomfort when asked to make judgments about others." The participants believed that the video contained excerpts from a counseling session that the couple attended. The cover story indicated that the participants should pay close attention to the conversation in anticipation of answering some questions about the couple's relationship. At this point, the participants were taken to separate rooms where they completed the rest of the experiment alone until the debriefing. After the video was over, the participants were randomly assigned to hear that one member of the couple had an affair or committed suicide. The participants then indicated which person they thought had the affair or committed suicide. After making their decision, the participants were randomly assigned to hear confirming feedback (i.e., "You're right. That is the person who had the affair [committed suicide].") or disconfirming feedback (i.e., "Actually, it was the other person who had the affair [committed suicide]."). Approximately $3 \mathrm{~min}$ after hearing postdecision feedback, the participants completed a two-page questionnaire. They answered questions about the event, the decision-making process, and their general abilities (see Table 1 for dependent measures). After completing the dependent measures questionnaire, the participants were thanked for their participation and debriefed. Because some participants might have been upset after having made a decision about which member of the pair committed suicide, the debriefing explained that the people on the tape were actors and that no one actually committed suicide. In addition, the debriefing gave participants the location and phone number of the university counseling center in case they were upset or anxious as a result of their participation. To our knowledge, no participant ever sought help from the counseling center as a result of participation in our experiment.

\section{Results}

All participants made a choice about which member of the couple committed suicide or had an affair. Thirtythree participants $(61.1 \%)$ in the suicide scenario condition believed that it was the man who committed suicide, and 26 participants $(59.1 \%)$ in the affair scenario condi- 
Table 1

Dependent Measures

Dependent Measures suicide], how certain were you that you chose the correct person?*

How much attention were you paying to the conversation the two people in the video were having?

At the time you decided which person had an affair [committed suicide], to what extent did you feel that you had a good basis (enough information) to make your decision?*

How easy or difficult was it for you to decide which person had an affair [committed suicide]?*

How long did it take you to decide which person had an affair [committed suicide]?*

In general, how good are you at making judgments about other people?

In general, how good are you at interpreting non-verbal behaviors?

Which statement describes your decision process? Choose one.

(a) As soon as the experimenter told me that one of the people had an affair [committed suicide], I knew exactly who it was.

(b) I had to think back about the conversation and reconstruct it before I could decide which person had an affair [committed suicide].

Indicate the extent to which the following statement describes your thoughts:

"While I was watching the people's conversation on the videotape, I had a suspicion that one of them might have had an affair [committed suicide]."

In general, how good are you at detecting variations in people's tone of voice?†

At the time you were watching the video, to what degree did you notice hostility in the couple's conversation? $\dagger$

At the time you were watching the video, to what degree did you notice that the couple was anxious? $\uparrow$

At the time you were watching the video, to what extent did you notice that either member of the couple was frustrated? $\uparrow$

To what extent did you notice supportive comments from either member of the couple? $\dagger$

This statement does not describe my thoughts at all.

to

This statement describes my thoughts extremely well.

very poor

to

excellent

I didn't notice any hostility.

to

I noticed intense hostility.

I didn't notice any anxiety. to

I noticed intense anxiety.

I didn't notice any frustration. to

I noticed intense frustration.

I noticed a lot of supportive comments. to

I didn't notice any supportive comments.

Think for a moment about the member of the couple who had the affair. I can't recall very many negative To what extent can you recall that person making negative [positive] [positive] comments. comments about the relationship or their partner? $\dagger$

to

I can recall a lot of negative

[positive] comments.

To what extent do you think the information you received about the accuracy of your judgment affected your answers to any of the questions you have just answered? $\uparrow$

It didn't affect my answers to any of the questions.

to

It affected my answers to all of

the questions.

Note-*Indicates questions shown to half of the participants in Experiment 2 before feedback was given. $†$ Indicates questions added in Experiment 2. Only the affair scenario was used in Experiment 2.

tion believed that it was the man who had an affair. A multivariate analysis of variance (ANOVA) revealed no interaction between feedback and the choice of male or female $[F(10,84)=1.12, p=.19]$. However, there was a significant main effect of the feedback variable and a significant feedback $\times$ story interaction $[F \mathrm{~s}(9,85)>$ 
$2.28, p s<.03]$. Subsequently, univariate ANOVAs were conducted to determine which dependent variables contributed to the significant omnibus effects.

The univariate ANOVAs revealed a main effect of feedback on six variables; confirming feedback inflated participants' reports of retrospective confidence in their decision, the basis for their decision, the ease with which the decision was made, the speed with which the decision was made, how good they are at making judgments about others, and how good they are at interpreting nonverbal behaviors (see Table 2). The main effect of feedback on participants' reports of ability to make judgments of others was qualified by a significant feedback $\times$ story interaction $[F(1,93)=11.39, p=.02]$. The effect of feedback was larger in the affair condition than in the suicide condition $[t(52)=2.10, p=.04$ and $t(42)=$ $1.26, p=.22$, respectively]. A second two-way interaction, on reports of attention $[F(1,93)=11.00, p=$ .03 ], produced the opposite pattern - the effect of feedback was larger in the suicide condition than in the affair condition $[t(42)=4.75, p<.01$ and $t(52)=1.19, p=$ .24 , respectively].

Participants indicated whether their decision was automatic or a result of a reconstruction process. A test for the equality of proportions revealed that participants who received confirming feedback were more likely to report that their decision was automatic than were participants who received disconfirming feedback (38\% vs. $11 \%, z=3.05, p<.05)$.

\section{Discussion}

The results of this experiment were consistent with the predictions: Confirming feedback inflated retrospective reports in comparison with disconfirming feedback. Recall that the dependent measures required participants to report on events that occurred before feedback was given. Because participants were randomly assigned to condition, their reports on the dependent measures questionnaire should have been equivalent across conditions. Instead, feedback distorted participants' recollections of prefeedback judgments about the event (e.g., reports of the basis for the decision), the decision process (e.g., reports of recalled certainty in the decision), and general abilities (e.g., reports of ability to interpret nonverbal be- havior). Because the feedback manipulation did not occur until after participants' decisions were made, the observed differences are due to the feedback manipulation rather than to any prefeedback differences across conditions.

These results are consistent with those observed in memory paradigms of hindsight research. In those paradigms, people misremember previous judgments or predictions once they learn outcome information (e.g., Fischhoff, 1977). The present research was designed so that participants could not misremember the prediction itself. Even so, they demonstrated substantial susceptibility to outcome information when asked to remember judgments related to their prediction. For example, participants learning that their decision was correct produced a host of responses consistent with having known the correct answer all along: remembering that they were confident when their decision was made, remembering that they had a good basis for their decision, and so forth. Interestingly, these people also inflated reports on variables consistent with being correct about these kinds of decisions in general: Confirming feedback inflated reports of ability to interpret nonverbal behaviors. Participants who received disconfirming feedback produced a similar pattern of responses except that theirs were consistent with having "known all along" that their answer was incorrect: retrospective certainty was low, the basis for the decision was reported to be poor, and so forth.

As noted in the introduction, these results have theoretical and practical importance. Practically, consider a situation in which self-reported certainty is used to assess decision accuracy. Evaluating an eyewitness is a good example of a situation in which the self-reported certainty of the decision maker (i.e., the eyewitness) is a critical determinant of whether the decision (i.e., the identification) is accepted as accurate. Indeed, self-reported certainty has been recognized as a critical variable by the U.S. Supreme Court (Neil v. Biggers, 1972) and is often used as an indicator of identification accuracy to the exclusion of other forensically relevant variables (e.g., Cutler, Penrod, \& Dexter, 1990). These results are theoretically important because they demonstrate that existing conceptualizations of the hindsight bias are too narrow: Outcome information still has a profound effect on retrospective judgments even when people cannot misremem-

Table 2

Means, Standard Deviations, Sample Sizes, and Effect Sizes for Dependent Measures in Experiment 1

\begin{tabular}{|c|c|c|c|c|c|c|c|c|c|c|c|c|c|c|}
\hline \multirow[b]{3}{*}{ Condition } & \multicolumn{14}{|c|}{ Dependent Measures } \\
\hline & \multicolumn{2}{|c|}{ Certainty } & \multicolumn{2}{|c|}{ Attention } & \multicolumn{2}{|c|}{ Basis } & \multicolumn{2}{|c|}{ Ease } & \multicolumn{2}{|c|}{ Long } & \multicolumn{2}{|c|}{ Judgments } & \multicolumn{2}{|c|}{ Nonverbals } \\
\hline & $M$ & $S D$ & $M$ & $S D$ & $M$ & $S D$ & $M$ & $S D$ & $M$ & $S D$ & $M$ & $S D$ & $M$ & $S D$ \\
\hline Confirming feedback & \multicolumn{2}{|c|}{$n=45$} & \multicolumn{2}{|c|}{$n=45$} & \multicolumn{2}{|c|}{$n=44$} & \multicolumn{2}{|c|}{$n=45$} & \multicolumn{2}{|c|}{$n=45$} & \multicolumn{2}{|c|}{$n=45$} & \multicolumn{2}{|c|}{$n=45$} \\
\hline Disconfirming feedback & $\begin{array}{r}46.41 \\
n\end{array}$ & $\begin{array}{l}22.63 \\
53\end{array}$ & $\begin{array}{c}8.49 \\
n=\end{array}$ & $\begin{array}{l}1.60 \\
53\end{array}$ & $\begin{array}{c}4.68 \\
n=\end{array}$ & $\begin{array}{l}3.59 \\
53\end{array}$ & $\begin{array}{c}6.91 \\
n=\end{array}$ & $\begin{array}{l}1.95 \\
53\end{array}$ & $\begin{array}{c}4.26 \\
n=\end{array}$ & $\begin{array}{l}2.19 \\
53\end{array}$ & $\begin{array}{c}6.07 \\
n=\end{array}$ & $\begin{array}{l}1.57 \\
53\end{array}$ & $\begin{array}{c}6.26 \\
n\end{array}$ & $\begin{array}{l}1.47 \\
53\end{array}$ \\
\hline $\begin{array}{l}p \\
d\end{array}$ & 1 & $\begin{array}{l}00 \\
07\end{array}$ & & & & $\begin{array}{l}00 \\
67\end{array}$ & & 20 & & $\begin{array}{l}70 \\
79\end{array}$ & & 0 & & 1 \\
\hline
\end{tabular}

Note-The values in the bottom row represent Cohen's $d$. 
ber their prior prediction. Research suggesting that people use feelings of certainty as a guide when evaluating outcome information (e.g., Werth \& Strack, 2003) should take these results into consideration: Feelings of certainty (as well as other variables) can themselves be distorted by outcome information.

One explanation for the results observed here is that participants have not thought about the questions they are being asked before seeing them on the dependent measures questionnaire. There is no reason to expect participants to independently "record" their judgments on variables such as the basis for their decision, how much attention they paid, how easy their decision was, and so forth. Although participants may not consider these judgments at the time of their decision, they must consider them when called upon to answer the dependent measures questionnaire. Because the questionnaire is presented after feedback is given, participants cannot assess their confidence (or other judgments) in a feedbackfree environment. The only way they can consider these judgments is through the lens of the knowledge that their decision was correct (or incorrect). The assumption is that this lens distorts their memories to make them consistent with the newly acquired outcome information; that is, "I must have paid close attention, I got the right answer." If this interpretation is correct, ensuring that participants consider judgments before they hear feedback should mitigate the effect of feedback. Without having outcome information to which retrospective judgments must conform, participants should produce equivalent judgments in the disconfirming and confirming feedback conditions. Experiment 2 was designed to test whether participants can be inoculated against postdecision feedback by thinking about some judgments before hearing feedback.

\section{EXPERIMENT 2}

Experiment 2 was designed to test the possibility that participants are affected by outcome information because they have not considered the judgments they are asked to make before outcome information is delivered. To test this possibility, participants were randomly assigned to see some of the questions to be asked in the dependent measures questionnaire (i.e., the "questionknowledge" manipulation), prior to receiving feedback (cf. Wells \& Bradfield, 1999). Participants were not asked to publicly indicate an answer to any question. Instead, they were directed to think privately about how they would answer the questions and were instructed that they would have an opportunity to answer them shortly. It was predicted that this manipulation would moderate the effect of postdecision feedback such that participants in the question-knowledge condition would display a smaller feedback effect than would participants in the no-question-knowledge condition.

\section{Method}

Participants and Design. The participants $(N=128$; 96 female $)$ were undergraduate students at Iowa State University. They were given extra credit in their psychology classes in exchange for their participation. The participants were randomly assigned to view (or not view) some of the dependent measures before feedback was given (the question-knowledge manipulation). In addition, the participants were randomly assigned to hear confirming or disconfirming feedback about the accuracy of an interpersonal judgment they made. The resulting design was a 2 (confirming feedback vs. disconfirming feedback) $\times 2$ (question-knowledge vs. no-question-knowledge) fully randomized, between-participants factorial.

Materials and Procedure. The procedure for Experiment 2 was identical to that for Experiment 1 with the following exceptions. First, recall that the suicide and affair scenarios had very similar effects on participants' reports. Therefore, only the affair scenario is used in Experiment 2 in order to reduce the possibility that a participant would become upset by having to make a judgment about which person committed suicide. The second change in Experiment 2 was that participants were randomly assigned to the questionknowledge or no-question-knowledge condition. The participants in the former condition received a sheet on which four of the dependent measures questions were listed (see Table 1). They were instructed to think privately about how they would answer those questions. The participants in the no-question-knowledge condition did not receive any information about the questions to be asked in the dependent measures questionnaire. After $4 \mathrm{~min}$ had passed, the participants were randomly assigned to receive confirming feedback (i.e., "I wanted to let you know that I noticed that you were right in your choice of which person had the affair.") or disconfirming feedback (i.e., "I wanted to let you know that I noticed that the person you chose was actually not the one who had the affair. It was the other person who had an affair."). Two minutes after hearing feedback, the participants completed a dependent measures questionnaire. The same questions used in Experiment 1 were used in Experiment 2 except for the addition of eight questions (see Table 1). These dependent measures were added to further explore the breadth of the postdecision feedback effect. After completing the dependent measures questionnaire, the participants were thanked for their participation, debriefed, and dismissed.

\section{Results}

As in Experiment 1, all participants made a choice about which member of the couple had an affair. Seventysix participants $(57.6 \%)$ believed that it was the man who had an affair. As in Experiment 1, a multivariate ANOVA revealed no interaction between feedback and choice of male or female $[F(16,113)=0.45, p=.96]$. In order to determine whether the question-knowledge manipulation moderated the effect of postdecision feedback, a multivariate two-way ANOVA was conducted using feedback and question-knowledge as independent variables. This analysis revealed a significant main effect of feedback and a significant feedback $\times$ question-knowledge interaction $[F(15,114)=2.61, p<.01$ and $F(15,114)=$ $2.00, p=.02$, respectively] (see Table 3 for means and individual statistics). Univariate ANOVAs revealed four significant feedback $\times$ question-knowledge interactions, each of which is reported below.

Retrospective certainty. Retrospective certainty reports were inflated by confirming feedback in the noquestion-knowledge condition, compared with disconfirming feedback $[F(1,63)=6.92, p=.01]$ (see Figure 1). However, in the question-knowledge condition, feedback did not affect certainty reports $[F(1,65)=0.21, p=.65]$. This interaction pattern confirms the hypothesis that knowledge about which questions would be asked (i.e., 







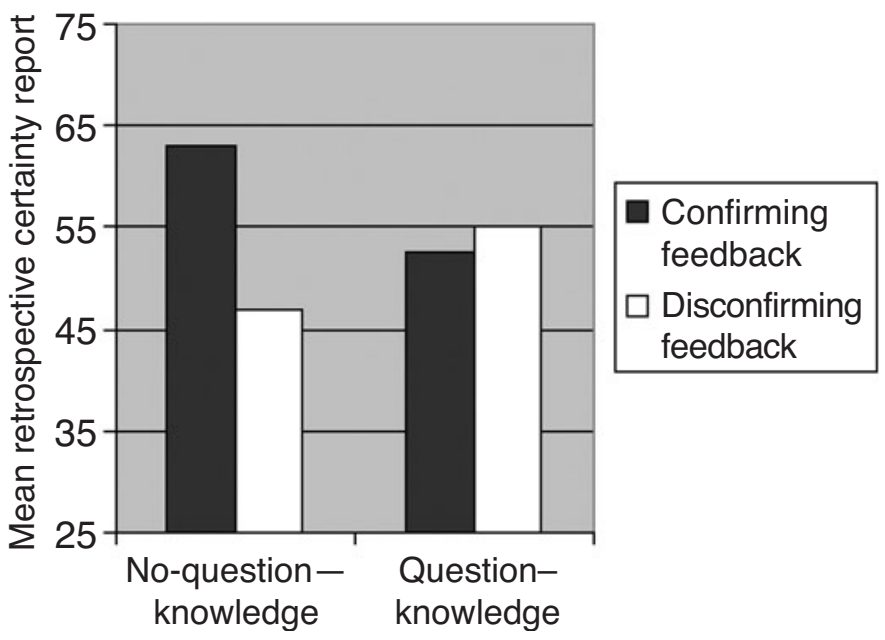

Figure 1. Feedback $\times$ question-knowledge interaction on reports of retrospective certainty.

the question-knowledge manipulation) would mitigate the effect of postdecision feedback. Presumably, this occurred because participants in the no- question-knowledge condition did not consider their confidence before hearing outcome information about the accuracy of their decision. In contrast, participants in the question-knowledge condition considered their confidence before hearing feedback, establishing a judgment independent of feedback, one they could access when answering the dependent measures questionnaire.

Ability to detect variations in tone of voice. Participants' reports of how able they were, in general, to detect variations in tone of voice produced a pattern of data similar to that reported above for retrospective certainty; the question-knowledge manipulation moderated the effect of feedback such that feedback had no effect in the question-knowledge condition $[F(1,65)=0.01, p=.95]$. In contrast, feedback did affect participants' reports in the no-question-knowledge condition: Compared with disconfirming feedback, confirming feedback inflated participants' reports of how able they were, in general, to detect variations in people's tone of voice $[F(1,63)=$ $9.69, p<.01]$.

Degree to which feedback influenced reports. The pattern of the interaction between feedback and questionknowledge on participants' reports of the degree to which feedback influenced their judgments was similar to the two patterns previously reported, demonstrating that question-knowledge again moderated the effect of feedback. There was a marginally significant effect of feedback in the no-question-knowledge condition: Participants who received confirming feedback acknowledged more influence of the feedback than did participants who received disconfirming feedback $[F(1,63)=3.06, p=$ $.09]$. In the question-knowledge condition, there was no effect of feedback $[F(1,65)=1.22, p=.27]$.
Noticing negative comments. Participants' reports of how many negative comments they noticed produced an interaction pattern different from those described above. For this dependent measure, although the questionknowledge manipulation moderated the effect of feedback, the moderation pattern was the opposite of what was predicted: In the no-question-knowledge condition, feedback did not affect participants' reports $[F(1,63)=$ $1.72, p=.20]$. In contrast, in the question-knowledge condition, participants who received confirming feedback reported recalling more negative comments than did those who received disconfirming feedback $[F(1,65)=$ $3.79, p=.05]$.

Main effects of feedback. In addition to the interactions between feedback and question-knowledge described above, there were four main effects of feedback: Participants who received confirming feedback reported having a greater basis on which to make their decision, making their decision more quickly, being better at making judgments about other people, and noticing fewer supportive comments from either member of the couple, $F \mathrm{~s}(1,127)>4.18, p \mathrm{~s}<.04]$. There was one marginally significant effect: Compared with participants who heard disconfirming feedback, those who heard confirming feedback reported making their decision more easily $[F(1,131)=2.94, p=.09]$.

\section{Discussion}

The postdecision feedback effect was replicated in Experiment 2: Confirming feedback inflated participants' reports of the basis they had for their decision, the length of time it took to make their decision, the certainty with which the decision was made, the ease with which the decision was made, and the extent to which they are good at making judgments about others in general. Confirming feedback also inflated reports on two dependent mea- 
sures that were new to Experiment 2. First, confirming feedback inflated participants' reports of how good they are, in general, at detecting variations in people's tone of voice. Second, confirming feedback caused participants to report noticing fewer supportive comments from either member of the couple. These results are consistent with the notion that postdecision feedback influences a broad range of dependent measures, including those that are related to general abilities and observations, rather than details of the video or decision.

Beyond replicating the effects of Experiment 1, in Experiment 2 we tested the hypothesis that the postdecision feedback effect is due to participants' failure to consider judgments related to their decision until they are presented with the dependent measures. Therefore, half of the participants were randomly assigned to see some questions they would be asked before they heard outcome information. The other half of the participants did not see the questions they would be asked. Results indicated that the question-knowledge manipulation mitigated the effect of feedback on three dependent measures: participants' reports of their certainty at the time of their decision, their general ability to notice variations in people's tone of voice, and their reports of the extent to which the feedback influenced their answers. In addition, although the interactions did not achieve significance, the same pattern of means was obtained on participants' reports of how easy the decision was and how long their decision took, both of which are questions that participants were instructed to consider in the question-knowledge condition. ${ }^{2}$ These patterns support the hypothesis that participants are affected by outcome information because they do not independently consider judgments related to the video or their decision before hearing feedback. Once feedback is given, participants can only respond to the dependent measures questionnaire in the context of the knowledge that their decision was correct or incorrect.

Evidence that the question-knowledge manipulation mitigated the postdecision feedback effect also indicates that participants were not taking advantage of an opportunity to present themselves as skilled decision makers (i.e., the results are inconsistent with a motivational explanation). In particular, the pattern of responses is not consistent with one strategy for maximizing positive impressions: augmentation (Kelley, 1973). Kelley's augmentation principle states that people make internal attributions for success when a successful outcome is achieved in the presence of external barriers. Therefore, participants would have had to emphasize the external barriers to success (e.g., the lack of information on which to base a decision) to take advantage of the opportunity for augmentation. For example, participants who received confirming feedback could have reasoned: "I made the right choice in spite of the fact that there wasn't much information on which to base my decision." Instead, participants in the confirming feedback condition acknowledged external variables that facilitated their ability to make a correct decision; those who received confirming feedback indicated that they had a better basis for making their decision than did people who received disconfirming feedback.

Similarly, if participants in the disconfirming feedback condition are motivated to preserve their image as good decision makers, they should report an even poorer basis for their decision, an even more difficult time making the decision, even less certainty, and so forth in the questionknowledge condition than in the no-question-knowledge condition. Such reports would at least make them appear consistent in the face of having made an inaccurate decision and preserve the possibility that they are skillful decision makers in other contexts. In general, these results are consistent with other research on the hindsight bias, suggesting that motivational explanations for the bias are not sufficient (e.g., see Hawkins \& Hastie, 1990; Hell et al., 1988).

Although the question-knowledge manipulation only moderated the feedback effect for three dependent measures, we believe that the obtained results support the conclusion that the postdecision feedback effect is not driven by participants' motivation to look like good decision makers. This is because the pattern appears on what is arguably the most relevant dependent measure: retrospective certainty. Theoretical research on the hindsight bias reveals that subjective feelings of certainty are a key determinant of how people will react to outcome information (e.g., Werth \& Strack, 2003; Werth, Strack, $\&$ Förster, 2002). For example, Werth and Strack demonstrated that the hindsight bias was larger for judgments originally made with high confidence than it was for judgments originally made with low confidence.

The results reported here are consistent with the notion that feedback affects retrospective judgments automatically. Future research will be needed to clarify what the nature of that automatic effect is. For example, is it true that participants fail to consider the judgments before seeing the questionnaire or are previously considered judgments rendered inaccessible by feedback? Alternatively, does feedback actually alter memories of the videotaped event and decision process? Future research investigating the automaticity of the feedback effect might include a control condition in which participants hear no information about their accuracy. This condition would be important to establish a record of judgments in the absence of any information about accuracy. Unpublished data indicate that reports on the decision processes in a control condition fall between the means observed for disconfirming and confirming feedback conditions, with the means in the control condition significantly different from the confirming feedback condition more often than from the disconfirming feedback condition (Bradfield, 2001, Experiment 3). The details of that experiment are not reported here because the effects of two new manipulations (cognitive load and time pressure) were largely uninterpretable. If future research does determine that the effect of feedback alters memo- 
ries, it will be consistent with the explanation Fischhoff (1975) originally offered for the hindsight bias.

\section{GENERAL DISCUSSION}

The two experiments reported here provide evidence on how outcome information affects retrospective reports when people cannot misremember a prior decision. Experiment 2 explored one way to mitigate the effect of outcome information in a social judgment paradigm: the question-knowledge manipulation. Other research on the hindsight bias indicates that the magnitude of the effect is also reduced when people consider alternatives to their chosen prediction (e.g., Arkes, Faust, Guilmette, \& Hart, 1988; Carli \& Leonard, 1989; Slovic \& Fischhoff, 1977). In the present research, participants chose which member of a couple had an affair. Perhaps the magnitude of postdecision feedback effect would be reduced if participants were required to consider information consistent with the nonchosen partner having an affair or commiting suicide. We would predict a reduction in the magnitude of the hindsight bias only if participants were asked to consider few pieces of information consistent with the nonchosen partner having an affair; instructions to consider many reasons for an alternative outcome do not reduce the hindsight bias, presumably because thinking of many alternatives is difficult (Sanna, Schwarz, \& Stocker, 2002).

Is it optimal to reduce or eliminate the hindsight bias? One argument in favor of trying to reduce the hindsight bias is that distorted retrospective assessments of decision processes may affect future decisions. For example, suppose doctors learn that a diagnosis they have made is correct. On the basis of the research reported here, we would expect confirming outcome information to inflate doctors' retrospective certainty, their memories of how easy their diagnosis was, and maybe even their feelings of how good their general diagnostic ability is. What are the implications of those distortions for future decisions? If doctors recall being very certain in a diagnosis and recalls that it was made easily, will that give them a distorted sense of their diagnostic ability? Will it cause them to overestimate their ability to make diagnoses that are beyond their expertise (cf. Bukszar \& Connolly, 1988)? Future research should address this question of whether outcome information about prior decisions affects subsequent judgments or decision processes.

Although outcome information can potentially have negative implications on cognitive functioning, it is important to acknowledge the potential adaptive consequences of outcome information. For example, outcome information helps people improve judgmental accuracy when decisions are easy (Hoch \& Loewenstein, 1989). In addition, it seems that people could benefit from the memory distortions illustrated in these experiments by using them as a means to justify a decision to themselves (e.g., Baumeister, 1998). For example, could failure to incorporate distorted recollections into a coherent selfschema have implications for mental well-being? Re- search indicates that people who feel positively about their partner in a relationship recall more positive aspects of the relationship than do people who feel negatively, suggesting that distorted recollections may serve a cognitive benefit (Halford, Keefer, \& Osgarby, 2002). Future research should explore the potentially adaptive side of the hindsight bias.

The experiments reported here demonstrate effects of outcome information beyond what has been traditionally observed in hindsight research. In the present experiments, outcome information distorted the recollections related to a decision rather than recollections of the decision itself. In addition, these distortions covered a broad range of judgments related to the decision, rather than simply a subjective probability assessment or answer to a general knowledge question. The second experiment provides evidence suggesting that the results obtained are consistent with an automatic explanation for the effect (cf. Fischhoff, 1975). Future research will be needed to confirm the precise nature of this phenomenon's automaticity. In the meantime, this research suggests that we should use caution when evaluating the recollections surrounding a social decision; people's recollections are not reliable sources of information about their own prior decision-making processes.

\section{REFERENCES}

Arkes, H. R., Faust, D., Guilmette, T. J., \& Hart, K. (1988). Eliminating the hindsight bias. Journal of Applied Psychology, 73, 305-307.

Arkes, H. R., Wortmann, R. L., Saville, P. D., \& Harkness, A. R. (1981). Hindsight bias among physicians weighing the likelihood of diagnoses. Journal of Applied Psychology, 66, 252-254.

Baboushin, H. R., Hardoon, K. K., Derevensky, J. L., \& Gupta, R. (2001). Underlying cognitions in gambling behavior among university students. Journal of Applied Social Psychology, 37, 1409-1430.

Baumeister, R. F. (1998). The self. In D. Gilbert, S. Fiske, \& G. Lindzey (Eds.), The handbook of social psychology (4th ed., pp. 680-740). New York: McGraw-Hill.

BRADFIELD, A. L. (2001). Bogus feedback distorts recall of an interpersonal judgment task: Three empirical tests of the post-decision feedback effect. Unpublished doctoral dissertation, Iowa State University.

Bukszar, E., \& Connolly, R. (1988). Hindsight bias and strategic choice: Some problems in learning from experience. Academy of Management Journal, 31, 628-641.

CAMPBELL, J. D., \& TESSER, A. (1983). Motivational interpretations of hindsight bias: An individual difference analysis. Journal of Personality, 51, 605-620.

CANNON, C. K., \& QUINSEY, V. L. (1995). The likelihood of violent behaviour: Predictions, postdictions, and hindsight bias. Canadian Journal of Behavioural Science, 27, 92-106.

CARLI, L. L. (1999). Cognitive reconstruction, hindsight, and reactions to victims and perpetrators. Personality \& Social Psychology Bulletin, 25, 966-979.

CARLI, L. L., \& LeONARD, J. B. (1989). The effect of hindsight on victim derogation. Journal of Social \& Clinical Psychology, 8, 331-343.

Casper, J. D., Benedict, K., \& Kelly, J. R. (1988). Cognitions, attitudes, and decision-making in search and seizure cases. Journal of Applied Social Psychology, 18, 93-113.

Christensen-Szalanski, J. J., \& Willham, C. F. (1991). The hindsight bias: A meta-analysis. Organizational Behavior \& Human Decision Processes, 48, 147-168.

Cutler, B. L., PenroD, S. D., \& Dexter, H. R. (1990). Juror sensitivity to eyewitness identification evidence. Law \& Human Behavior, 14, 185-191.

FiscHHOFF, B. (1975). Hindsight $\neq$ foresight. Journal of Experimental Psychology: Human Perception \& Performance, $1, \underline{1}, 288-299$. 
Fischioff, B. (1977). Perceived informativeness of facts. Journal of Experimental Psychology: Human Perception \& Performance, $\mathbf{3}_{2}$ 349-358.

FischHOFF, B., \& BEYTH, R. (1975). "I knew it would happen"Remembered probabilities of once-future things. Organizational Behavior \& Human Performance, 13, 1-16.

HALFORD, W. K., KeEFER, E., \& OSGARBY, S. M. (2002). "How has the week been for you two?" Relationship satisfaction and hindsight memory biases in couples' reports of relationship events. Cognitive Therapy \& Research, 26, 759-773.

Hastie, R., SchKade, D. A., \& PAYNe, J. W. (1999). Juror judgments in civil cases: Hindsight effects on judgments of liability for punitive damages. Law \& Human Behavior, 23, 597-614.

Hawkins, S. A., \& Hastie, R. (1990). Hindsight: Biased judgments of past events after the outcomes are known. Psychological Bulletin, 107, 311-327.

Hell, W., Gigerenzer, G., Gauggel, S., Mall, M., \& Müller, M. (1988). Hindsight bias: An interaction of automatic and motivational factors? Memory \& Cognition, 16, 533-538.

Hertwig, R., GigerenZer, G., \& Hoffrage, U. (1997). The reiteration effect in hindsight bias. Psychological Review, 104, 194-202.

Hoch, S. J., \& Loewenstern, G. F. (1989). Outcome feedback: Hindsight and information. Journal of Experimental Psychology: Learning, Memory, \& Cognition, 15, 605-619.

Hoffrage, U., Hertwig, R., \& Gigerenzer, G. (2000). Hindsight bias: A by-product of knowledge updating? Journal of Experimental Psychology: Learning, Memory, \& Cognition, 26, 566-581.

Hölzl, E., KIrChler, E., \& Rodler, C. (2002). Hindsight bias in economic expectations: I knew all along what I want to hear. Journal of Applied Psychology, 87, 437-443.

JANOFF-BulMAN, R., TIMKO, C., \& CARLI, L. L. (1985). Cognitive biases in blaming the victim. Journal of Experimental Social Psychology, 21, 161-177.

Kelley, H. (1973). The processes of causal attribution. American Psychologist, 28, 107-128.

LABINE, S. J., \& LABINE, G. (1996). Determinations of negligence and the hindsight bias. Law \& Human Behavior, 20, 501-516.

Louie, T. A., Curren, M. T., \& Harich, K. R. (2000). "I knew we would win": Hindsight bias for favorable and unfavorable team decision outcomes. Journal of Applied Psychology, 85, 264-272.

NeIL V. Biggers. 409 U.S. 188 (1972).

PoHL, R. F. (1998). The effects of feedback source and plausibility of hindsight bias. European Journal of Cognitive Psychology, 10, 191-212.

PoHL, R. F., \& HeLl, W. (1996). No reduction in hindsight bias after complete information and repeated testing. Organizational Behavior \& Human Decision Processes, 67, 49-58.

Pohl, R. F., Schwarz, S., Sczesny, S., \& Stahlberg, D. (2003). Hindsight bias in gustatory judgments. Experimental Psychology, 50, 107-115.

Robbennolt, J. K., \& Sobus, M. S. (1997). An integration of hindsight bias and counterfactual thinking: Decision-making and drug courier profiles. Law \& Human Behavior, 21, 539-560.
Roese, N. J., \& Maniar, S. D. (1997). Perceptions of purple: Counterfactual and hindsight judgments at Northwestern Wildcats football games. Personality \& Social Psychology Bulletin, 23, 1245-1253.

SANNA, L. J., SchWARZ, N., \& STOCKER, S. L. (2002). When debiasing backfires: Accessible content and accessibility experiences in debiasing hindsight. Journal of Experimental Psychology: Learning, Memory, \& Cognition, 28, 497-502.

SHARPE, D., \& ADAIR, J. G. (1993). Reversibility of the hindsight bias: Manipulation of experimental demands. Organizational Behavior \& Human Decision Processes, 56, 233-245.

SLOVIC, P., \& FISCHHOFF, B. (1977). On the psychology of experimental surprises. Journal of Experimental Psychology: Human Perception \& Performance, 3, 544-551.

Stallard, M., \& WORTHINGTON, D. L. (1998). Reducing the hindsight bias utilizing attorney closing arguments. Law \& Human Behavior, 22, 671-683.

WELLS, G. L., \& BRADFIELD, A. L. (1999). Eyewitnesses' recollections of their confidence, witnessing conditions, and identification decisions: The distorting effects of feedback. Psychological Science, 10, 138-144.

Werth, L., \& STRACK, F. (2003). An inferential approach to the knewit-all-along phenomenon. Memory, 11, 411-419.

Werth, L., Strack, F., \& Förster, J. (2002). Certainty and uncertainty: The two faces of the hindsight bias. Organizational Behavior \& Human Decision Processes, 87, 323-341.

Wood, G. (1978). The knew-it-all-along effect. Journal of Experimental Psychology: Human Perception \& Performance, 4, 345-353.

\section{NOTES}

1. Some researchers (e.g., Hertwig, Gigerenzer, \& Hoffrage, 1997) reserve the term hindsight bias for paradigms in which participants recall actual judgments (e.g., "memory" paradigms) and the "I knew it all along" effect for paradigms in which participants indicate how they would have judged, had they not known the outcome (e.g., "hypothetical" paradigms). The term "hindsight bias" as used here refers to the body of literature that encompasses both memory and hypothetical designs.

2. It is unclear why the effect of feedback on participants' reports of the basis for their decision was nearly identical in the question-knowledge and no-question-knowledge conditions. One possibility is that the basis question refers to an element of the video. The other three questions asked in the question-knowledge condition (certainty, ease, and length) concern aspects of the decision. Perhaps the benefit from questionknowledge, in terms of mitigating the effect of feedback on the questions included in the manipulation, only occurs for judgments related to a choice, rather than judgments related to a stimulus. Future research should investigate this possibility.

(Manuscript received June 19, 2003; revision accepted for publication May 7, 2004.) 